\title{
Modeling of Monopulse Radar Signals Reflected from Ground Clutter in a Time Domain Considering Doppler Effects
}

\author{
Jeong-Hun Nam ${ }^{1} \cdot J a e-W o n$ Rim $^{1} \cdot$ Hyunsoo Lee ${ }^{2} \cdot \mathrm{Il}^{-S e u k} \mathrm{Koh}^{1, *} \cdot$ Jong-Hwa Song ${ }^{3}$
}

\begin{abstract}
To evaluate the performance of a monopulse radar system, it is necessary to accurately model radar return signals from the ground surface in a time domain. In this paper, we propose a numerical method to model these return signals including radar radio frequency specifications, such as the pulse repetition interval, frequency, and polarization, as well as the antenna geometry, the ground clutter backscattering characteristics, and so on. The Doppler effect is also incorporated into the signal generation scheme because of the dynamics of the platform/clutter and the antenna orientation. The Doppler frequency shift caused by the ground clutter is modeled by employing the time correlation of the received signals. In some scenarios, the monopulse signals are generated and numerically examined. For real radar application, the effect of the platform's roll stabilization on the monopulse signals is investigated based on the proposed signal generation scheme.
\end{abstract}

Key Words: Doppler Effect, Ground Clutter, Monopulse Radar, Radar Return Signal.

\section{INTRODUCTION}

The radar system mounted on an airborne platform operates in an air-to-ground mode as well as in an air-to-air mode [1]. For example, the AN/APG-77 is the F-22's primary sensor that provides several functions, including terrain mapping, groundmoving target searching/tracking, and air-to-ground ranging (AGR) [2]. The AN/APG-77 radar adopts a monopulse system with an active electronically scanned array (AESA) antenna. The amplitude- or phase-comparison monopulse radar transmits one pulse and then receives three monopulse channels as the sum, elevation, and azimuth difference channels. The chan- nels are calculated as three weighted sums of partial signals received by each antenna element in a receiving mode [3, 4]. Also, in the air-to-ground mode, the clutter backscattering may become much stronger than that of the air-to-air mode, and such strong backscattering can seriously degrade the radar's performance [5].

The radar echo signals are determined by basic radar radio frequency $(R F)$ specification, such as frequency and pulse repetition interval (PRI), etc., and the radar's operation environment, such as platform dynamics, ground clutter type, antenna footprint on the ground plane (mainly determined by the platform orientation), and the ASEA antenna's beam illumination. Due

Manuscript received May 31, 2019 ; Revised October 1, 2019 ; Accepted May 12, 2020. (ID No. 20190531-044J)

${ }^{1}$ Deparment of Electronic Engineering, Inha University, Incheon, Korea.

${ }^{2}$ Agency for Defense Development, Daejeon, Korea.

${ }^{3}$ Hanwha Systems, Yongin, Korea.

"Corresponding Author: Il-Suek Koh (e-mail: ikoh@inha.ac.kr)

This is an Open-Access article distributed under the terms of the Creative Commons Attribution Non-Commercial License (http://creativecommons.org/licenses/by-nc/4.0) which permits unrestricted non-commercial use, distribution, and reproduction in any medium, provided the original work is properly cited.

(c) Copyright The Korean Institute of Electromagnetic Engineering and Science. All Rights Reserved. 
to the dynamics of the platform and ground clutter, the received signal is slightly distorted, which can be modeled by considering the Doppler frequency shift. Since the range-Doppler (RD) map clearly shows the Doppler frequencies, $\mathrm{RD}$-processing is crucial in many radar applications, including a synthetic aperture radar (SAR) [6] and a moving target indicator [7]. Hence, the modeling of the radar echo signal in a time-domain is required when considering the effect of all the aforementioned factor's needed to generate an accurate $\mathrm{RD}$ map.

Much attention has been paid to the backscattering determined by the ground clutter, which can be used to determine the mean power received by the radar. Since the clutter signal is a typical random signal, several probability density functions (PDF) have been used, such as Gaussian, Weibull, K-distribution, and so on [8]. Except in the case of sea clutter, the Gaussian distribution is a simple and accurate way to develop a time-domain signal model [9]. The correlated Gaussian random signal can be generated in a relatively easy and accurate manner $[10,11]$, and is a conventional method for incorporating the Doppler effect of the clutter into the modeling of pulseDoppler radar signals [12].

In Section II, we address the proposed modeling approach of the radar echo signal for a phase-comparison monopulse radar [3], considering the Doppler effects due to the dynamics of the platform and the ground clutter simultaneously. Section III presents the numerical results for operating scenarios that consider the platform dynamics and antenna beam steering direction, etc. Finally, an AGR simulation is examined for a real-world application.

\section{MODELING METHOD}

\section{Scenario and Coordinate Systems}

The dynamic scenario for the air-to-ground radar operation is shown in Fig. 1. An airborne platform moves at a constant velocity, $\vec{v}=v_{x} \hat{x}+v_{y} \hat{y}+v_{z} \hat{z}$, while repeatedly transmitting a signal to the ground surface at every PRI. Given that an antenna beam is illuminated toward the line of sight (LOS), the return signals may be reflected primarily from the main-lobe beam projected on the ground that is truncated by the half-power beam width (HPBW) for a numerical simulation. The return signals of multiple pulses are aligned along the fast time $(t)$ and the slow time $(\eta)$, generating 2D raw signals [13]. The mean reflection power from the ground clutter inside the projected main-lobe beam can be calculated using the backscattering coefficient [14]. Here, we assume the ground clutter to be a rough surface [15] for which a theoretical backscattering model, such as an Integral Equation Model (IEM) may be accurate [16] for two co-polarized scatterings. The return signal can be assumed to be a Gaussian random signal whose backscattering coefficient

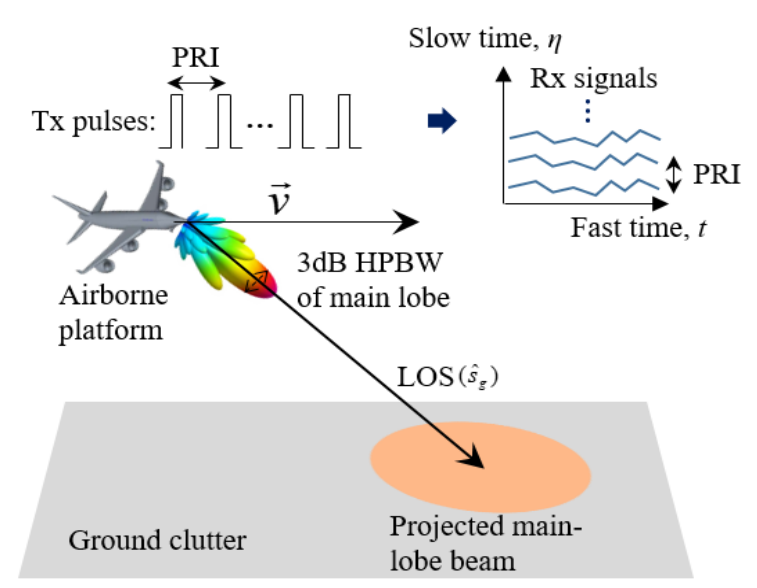

Fig. 1. Dynamics scenario for air-to-ground radar.

is used as a variance of the random signal. The movement of the platform and ground clutter can be modeled using the Doppler frequency shift in the received signals.

Note that the shape of the projected main-lobe beam in Fig. 1 is dependent on the orientations of the platform and the antenna. Hence, the coordinate systems of the platform and the antenna are important. The relationship between the global and local coordinate systems of the platform is shown in Fig. 2. The parameters related to the coordinate systems in Fig. 2 are summarized as follows:

\begin{tabular}{|c|c|}
\hline$\left(\hat{x}_{p}, \hat{y}_{p}, \hat{z}_{p}\right):$ & $\begin{array}{l}\text { Orientation vector of the platform in its local } \\
\text { coordinates, }\end{array}$ \\
\hline$\left(\hat{x}_{g}, \hat{y}_{g}, \hat{z}_{g}\right):$ & $\begin{array}{l}\text { Orientation vector of the platform in the global } \\
\text { coordinates, }\end{array}$ \\
\hline$\hat{s}_{l}$ and $\hat{s}_{g}:$ & $\begin{array}{l}\text { LOS vectors of the antenna in the platform's } \\
\text { local coordinates and in the global coordinates, } \\
\text { respectively, }\end{array}$ \\
\hline and $\varphi$ : & $\begin{array}{l}\text { Elevation and azimuth angles in the antenna's } \\
\text { local coordinates, }\end{array}$ \\
\hline${ }_{r}, \theta_{y}$, and $\theta_{p}$ : & $\begin{array}{l}\text { Roll, yaw, and pitch angles in the platform's } \\
\text { local coordinates. }\end{array}$ \\
\hline
\end{tabular}

As the local coordinate of the antenna coincides with that of the platform, $\hat{s}_{l}$ is defined as

$$
\hat{s}_{l}=\sin \theta \cos \varphi \hat{x}_{p}+\sin \theta \sin \varphi \hat{y}_{p}+\cos \theta \hat{z}_{p} .
$$

Considering the dynamics of the platform, the LOS vector of the antenna in the global coordinates is rotated depending on $\theta_{r}, \theta_{y}$, and $\theta_{p}$, as seen in Fig. 2. Based on $\theta_{r}, \theta_{y}$, and $\theta_{p}$, the antenna's LOS vector in the global coordinate, $\hat{s}_{g}$, is represented as

$$
\hat{s}_{g}=R_{x}\left(\theta_{r}\right) R_{y}\left(\theta_{y}\right) R_{z}\left(\theta_{p}\right) \hat{s}_{l}
$$

where $R_{x}, R_{y}$, and $R_{z}$ are rotation matrices along the global $x, y$, and $z$ axes, respectively [17]. In Fig. 3, the signs of the antenna weights for three monopulse channels are defined. The $V$ and $W$ 


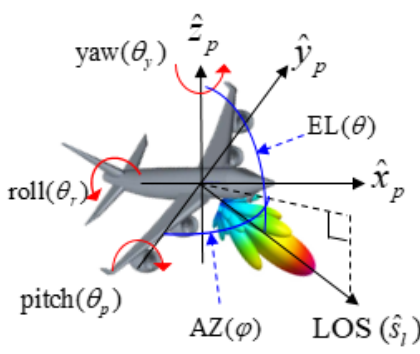

Platform's local coordinates

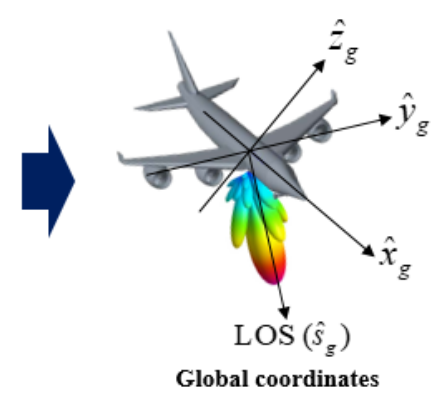

Fig. 2. Local and global coordinate systems of the platform and antenna.

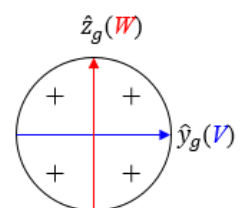

(a)

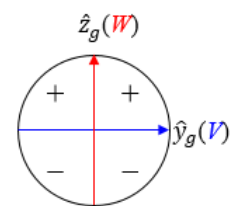

(b)

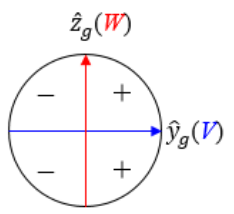

(c)
Fig. 3. Sign of antenna weight for phase-comparison monopulse sum and difference patterns: (a) sum $\left(w^{\Sigma}\right)$, (b) elevation difference $\left(w^{\Delta E L}\right)$, and (c) azimuth difference $\left(w^{\Delta A Z}\right)$. axes of the monopulse radar coincide with the $y_{g}$ and $z_{g}$ axes in the global coordinates, respectively [18]. In Figs. 4 and 5, the projected main-lobe beam and the $V$ and $W$ axes are found on the ground plane using a ray-tracing technique: first, the normal vector of the antenna's $V W$ plane is aligned in the direction of $\hat{s}_{g}$. Then, rays are launched from the antenna's center to the rim of the HPBW of the antenna pattern or the $V$ and $W$ lines in the antenna's $V W$ plane. Finally, the intercept points between the rays and the ground plane are calculated.

The simulation parameters are presented in Table 1. In Fig. 4, the platform orientation and antenna beam steering are given as $\theta_{r}=0^{\circ}, \theta_{y}=0^{\circ}, \theta_{p}=0^{\circ}$, and $\theta=105^{\circ}, \varphi=0^{\circ}-60^{\circ}$, respectively. As observed in Fig. 4(a), it is well-known that as the azimuth angle $(\varphi)$ increases, the HPBW of the $V$ axis broadens while the HPBW of the $W$ axis remains constant [19]. The simulation and theoretical estimations for the HPBW of the $V$ and $W$ axes on the ground are compared in Fig. 4(b), where an excellent agreement can be observed. As shown in Fig. 5 (a), if the platform is rolled, the monopulse axes rotate by a small angle, $\gamma$, with respect to the major axes parallel to the

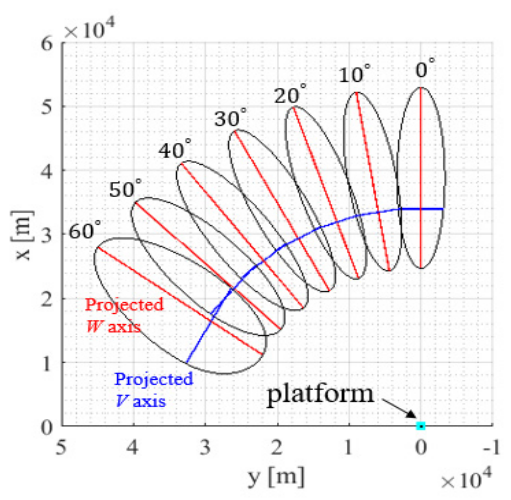

(a)

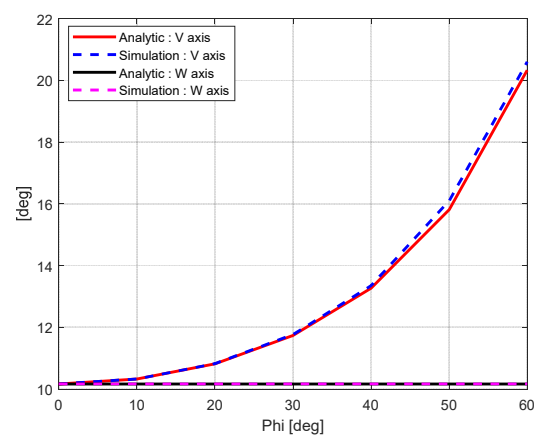

(b)

Fig. 4. Simulation results according to the azimuth angle $\varphi$. (a) Projected antenna footprint and $V$ and $W$ axes, and (b) analytic and simulation results of the $V$ and $W$ axis widths.

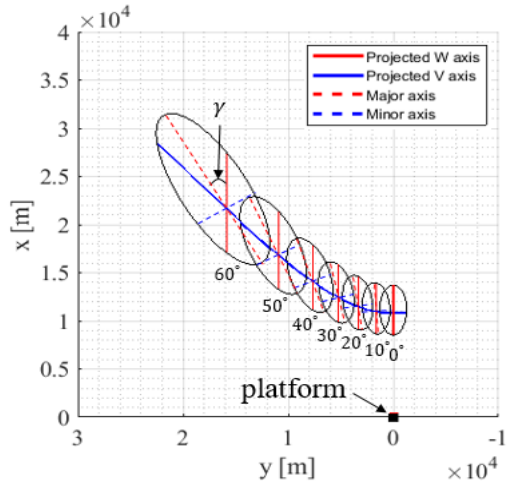

(a)

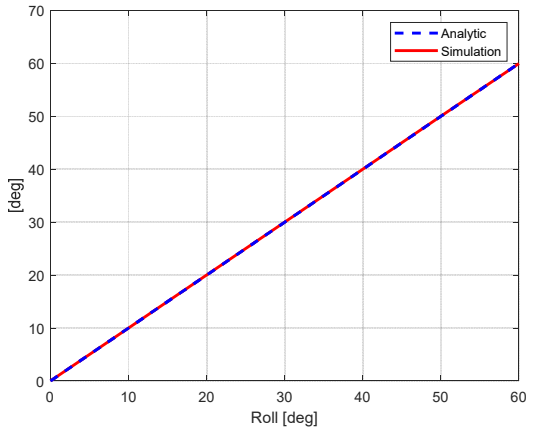

(b)

Fig. 5. Simulation results according to the rolling angle $\theta_{r}$. (a) Projected antenna footprint and the $V$ and $W$ axes, and (b) analytic and simulation results of $\gamma$. 
Table 1. Simulation parameters

\begin{tabular}{lc}
\multicolumn{1}{c}{ Parameter } & Value \\
\hline Polarization & $\mathrm{VV}$ \\
Frequency & $\mathrm{X}$ band \\
Number of pulses & 50 \\
Platform height $(\mathrm{feet})$ & 30,000 \\
Platform speed $(\mathrm{m} / \mathrm{s})$ & 180 \\
PRF $(\mathrm{Hz})$ & 1,670 \\
Rectangle size $(\mathrm{m})$ & $30 \times 30$ \\
Number of antenna array & \\
$V$ axis & 20 \\
$W$ axis & 20 \\
Antenna array spacing & \\
$V$ axis & $\lambda / 4$ \\
$\quad W$ axis & $\lambda / 4$ \\
Correlation function & Gaussian $[23]$ \\
Correlation length $(\mathrm{cm})$ & $8.4[5]$ \\
rms height $(\mathrm{cm})$ & $0.4[5]$ \\
Dielectric constant & $15.4-j 2.15[5]$ \\
\hline
\end{tabular}

LOS vector [14]. The estimations of $\gamma$ by simulation and by the known theoretical formula [14] are in excellent agreement, as seen in Fig. 5(b). For this calculation, the platform orientation angles are assumed as $\theta_{r}=0^{\circ}-60^{\circ}, \theta_{y}=0^{\circ}$, and $\theta_{p}=0^{\circ}$. The beam illumination angles of the antenna are fixed at $\theta=130^{\circ}$ and $\varphi=0^{\circ}$. Therefore, the projected antenna footprint on the ground plane can be accurately found for any platform and antenna orientation.

\section{Radar Return Signal in a Time-Domain}

The configuration of the signal transmission/reception at the radar and the corresponding projected main-lobe beam needed to calculate the radar return signals in a time-domain are shown in Fig. 6. First, the projected main-lobe beam is divided into uniform rectangular grids (cells) based on a preset range resolution. The range resolution is determined using the sampling time interval of the fast time $(t)$ [13]. The projected area can be approximated as an ellipse [20] whose major axis is parallel to

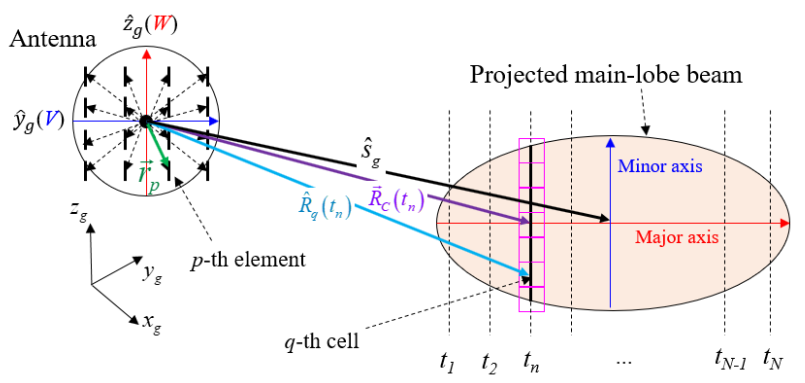

Fig. 6. Signal transmission/reception of antenna to/from surfaceprojected main-lobe beam. the LOS direction from the radar to the center of the ellipse. Since the minor axis is perpendicular to the major axis, the ellipse can be uniformly discretized using a rectangle along the major and minor axes, as seen in Fig. 6. The ellipse is located in the far-field region from the platform, and so the return signals from the cells along a line parallel to the minor axis are coherently added into one time-domain response in each sampling time (range bin). The received monopulse signals from each $q^{-}$ th ground cell is represented as

$$
\begin{aligned}
& \Psi_{q}^{\Sigma}\left(t_{n}, \eta\right)=\frac{A F_{\Sigma} A F_{\Sigma} G^{2} \sigma_{q}\left(t_{n}, \eta\right) A(q)}{R_{c}^{2}\left(t_{n}, \eta\right)} \delta\left[t-\frac{2 R_{c}\left(t_{n}, \eta\right)}{c}\right], \\
& \Psi_{q}^{\Delta E L}\left(t_{n}, \eta\right)=\frac{A F_{\Sigma} A F_{\Delta E L} G^{2} \sigma_{q}\left(t_{n}, \eta\right) A(q)}{R_{c}^{2}\left(t_{n}, \eta\right)} \delta\left[t-\frac{2 R_{c}\left(t_{n}, \eta\right)}{c}\right], \\
& \Psi_{q}^{\Delta A Z}\left(t_{n}, \eta\right)=\frac{A F_{\Sigma} A F_{\Delta A Z} G^{2} \sigma_{q}\left(t_{n}, \eta\right) A(q)}{R_{c}^{2}\left(t_{n}, \eta\right)} \delta\left[t-\frac{2 R_{c}\left(t_{n}, \eta\right)}{c}\right],
\end{aligned}
$$

where $\sigma_{q}$ and $A(q)$ are the backscattering coefficient and area of the $q$-th cell, respectively, $G$ is the gain of the antenna element, and $\delta(\cdot)$ is the Dirac delta function. Note that the $q$-th cell belongs to the sampling time $\left(t_{n}\right)$, as shown in Fig. 6. The sampling time can be computed simply as $t_{n}=2 R_{c}\left(t_{n}, \eta\right) / c$, where $c$ is the speed of light. $R_{c}\left(t_{n}, \eta\right)$ is the distance from the platform to the $q$-th cells corresponding to the major axis, as shown in Fig. 6. $A F_{\Sigma}, A F_{\triangle E L}$, and $A F_{\triangle A Z}$ are the array factors of the antenna for the sum, elevation difference, azimuth difference channels, respectively, on the $q$-th cell, which is given as

$$
\begin{aligned}
& A F_{\Sigma}=\sum_{p=1}^{N_{p}} w_{p}^{\Sigma} \exp \left(-j k_{0}\left[\hat{R}_{q}\left(t_{n}, \eta\right)-\hat{s}_{g}\right] \cdot \vec{r}_{p}\right), \\
& A F_{\Delta E L}=\sum_{p=1}^{N_{p}} w_{p}^{\Delta E L} \exp \left(-j k_{0}\left[\hat{R}_{q}\left(t_{n}, \eta\right)-\hat{s}_{g}\right] \cdot \vec{r}_{p}\right), \\
& A F_{\triangle A Z}=\sum_{p=1}^{N_{p}} w_{p}^{\Delta A Z} \exp \left(-j k_{0}\left[\hat{R}_{q}\left(t_{n}, \eta\right)-\hat{s}_{g}\right] \cdot \vec{r}_{p}\right),
\end{aligned}
$$

where $N_{p}$ is the number of the antenna elements and $k_{0}$ is the free-space propagation constant. $\hat{R}_{q}\left(t_{n}, \eta\right)$ is the direction vector from the phase center of the antenna to the $q$-th cell at $t=t_{n} \cdot \vec{r}_{p}$ is the displacement vector from the phase center of the antenna to the $p$-th antenna element. $w_{p}^{\Sigma}, w_{p}^{\Delta E L}$, and $w_{p}^{\triangle A Z}$ are the weight of the $p$-th antenna element for three monopulse channels, as seen in Fig. 3. In monopulse systems, a sum channel is used at the transmitting ( $\mathrm{Tx})$ mode and three channels, such as the sum, elevation difference, and azimuth difference channels, are employed in the receiving $(\mathrm{Rx})$ mode [4]. The total return signals sampled at $t=t_{n}$ are a summation of the return signal from specific ground cells. The calculated cells at $t=t_{n}$ are mathematically denoted as $\operatorname{Cell}(q) \in t_{n}$. Adding all responses from the ground cells belonging to 
$\operatorname{Cell}(q) \in t_{n}$, three monopulse signals can be formulated without the consideration of the Doppler effects as

$$
\Psi_{\Sigma, \Delta E L, \Delta A Z}\left(t_{n}, \eta\right)=\sum_{\text {Cell }(q) \in t_{n}}^{\text {Terrain }} \Psi_{q}^{\Sigma, \Delta E L, \Delta A Z}\left(t_{n}, \eta\right)
$$

where $\Psi_{\Sigma}, \Psi_{\triangle E L}$, and $\Psi_{\triangle A Z}$ are the total radar return signals for the monopulse sum, elevation difference, and aizumuth difference channels, respectively.

In Fig. 7, the return signal is calculated from a flat ground whose backscattering is assumed to be a constant at a slow time, $\eta=0$. In Fig. 7(a) and 7(b), the platform orientation and antenna beam steering are assumed as $\theta_{r}=60^{\circ}, \theta_{y}=0^{\circ}, \theta_{p}=$

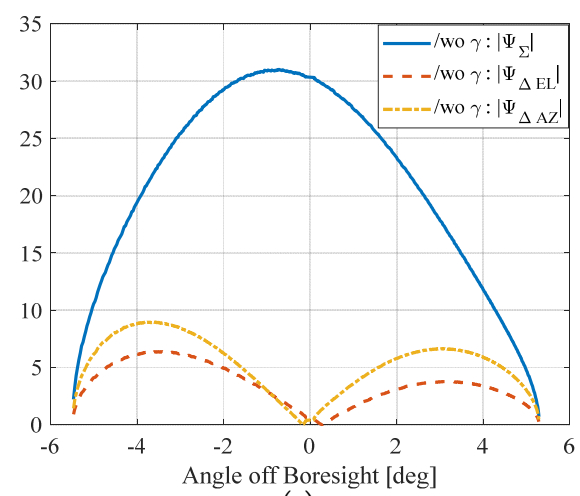

(a)

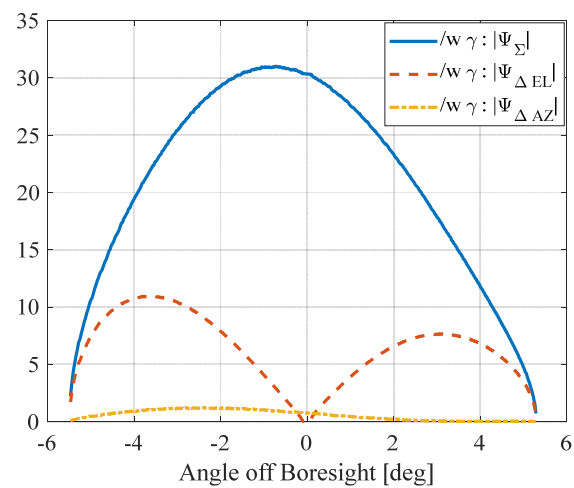

(b)

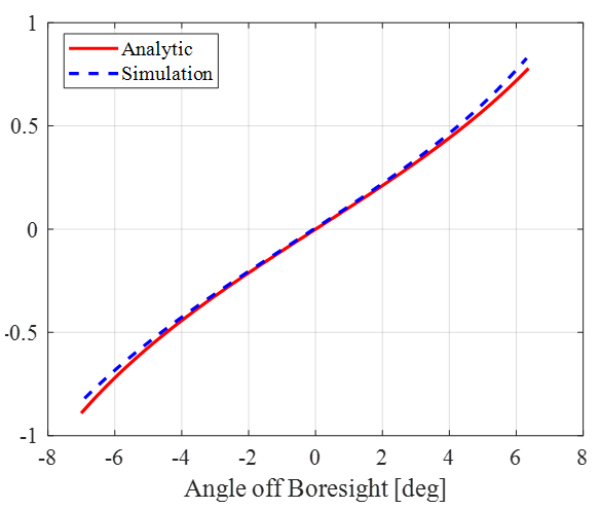

(c)

Fig. 7. Monopulse response vs angle-off boresight: (a) monopulse signal before $\gamma$-correction, (b) monopulse signal after $\gamma$ correction, and (c) monopulse ratio of $\operatorname{Im}\left(\Psi_{\triangle E L}\right)$ to $\operatorname{Re}\left(\Psi_{\Sigma}\right)$. $0^{\circ}$, and $\theta=130^{\circ}, \varphi=0^{\circ}$, respectively. The "/w $\gamma$ " and "/wo $\gamma$ " indicate "with $\gamma$-correction" and "without $\gamma$-correction", respectively. In Fig. 7(a), the roll angle is not zero, so it can be observed that the null of $\Psi_{\triangle E L}$ locates off the boresight and the monopulse response of $\Psi_{\triangle A Z}$ is not zero. But, as shown in Fig. $7(b)$, when the return signal is compensated with the known $\gamma$ correction scheme, the null point of $\Psi_{\triangle E L}$ is shifted to the boresight and the level of $\Psi_{\triangle A Z}$ is decreased to almost zero [4]. The exact and simulated monopulse ratios, $\operatorname{Im}\left(\Psi_{\Delta E L}\right) / \operatorname{Re}\left(\Psi_{\Sigma}\right)$, are compared in Fig. 7(c) for $\theta_{r}=0^{\circ}$ [14]. The proposed signal-generation scheme can provide an accurate monopulse ratio. The $\operatorname{Re}\left(\Psi_{\Sigma}\right)$ is approximately $2 \times 10^{-19}$, which is essentially zero. Here, $\operatorname{Re}(z)$ and $\operatorname{Im}(z)$ are the real and imaginary parts of $z$, respectively. Therefore, the generated time-domain signal can provide accurate monopulse signal behaviors.

\section{Doppler Effects}

There are two dominant Doppler effects in the dynamic situation considered here because of to the movements of the platform and ground clutter. The Doppler shift of the clutter can be included simply by generating the correlated Gaussian random variable of $\sigma_{q}\left(t_{n}, \eta\right)$ in the slow time in (1) for a proper correlation function, such as the Gaussian and exponential functions. Here, the Cholesky decomposition method is used [11]. The correlation time $\left(t_{c}\right)$ is inversely proportional to the Doppler frequency of the ground clutter [21, 22]. After computing (1) with the correlated $\sigma_{q}\left(t_{n}, \eta\right)$ along the slow time, the Doppler shift due to the platform dynamic is considered. The time-dependent distance between the platform and the $q$-th cell is expressed at the slow time $\left(\eta_{m}\right)$ as

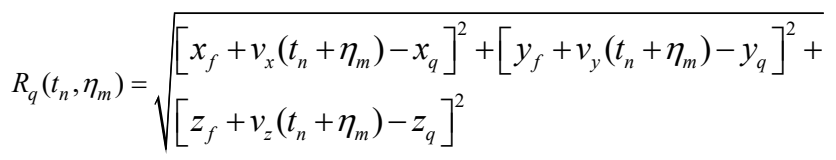

where $\left(x_{f}, y_{f}, z_{f}\right)$ and $\left(x_{q}, y_{q}, z_{q}\right)$ are the coordinates of the platform and the center of the $q$-th cell at $(t=0, \eta=0)$ of the global coordinates, respectively. By using the Taylor series expansion, the Doppler frequencies along the fast-time, $f_{D, t_{n}}$, and slow-time, $f_{D, \eta_{m}}$ axes, can be formulated as

$$
f_{D, t_{n}}=\frac{-\vec{R}_{q}\left(0, \eta_{m}\right) \cdot \vec{v}}{R_{q}\left(0, \eta_{m}\right) \lambda_{0}}, f_{D, \eta_{m}}=\frac{-\vec{R}_{q}(0,0) \cdot \vec{v}}{R_{q}(0,0) \lambda_{0}}
$$

where $\lambda_{0}$ is the free-space wavelength. Finally, the total return signal, including the Doppler effect due to platform movement, is given as

$$
\Psi_{\Sigma, \Delta E L, \Delta A Z}\left(t_{n}, \eta_{m}\right)=\sum_{\text {Cell }(q) \in t_{n}}^{\text {Terrain }}\left[\begin{array}{c}
\Psi_{q}^{\Sigma, \Delta E L, \Delta A Z}\left(t_{n}, \eta\right) \times \\
\exp \left(-j 2 \pi f_{D, t_{n}} t_{n}\right) \times \\
\exp \left(-j 2 \pi f_{D, \eta_{m}} \eta_{m}\right)
\end{array}\right] .
$$


If two Doppler effects are considered at the same time, it is physically expected that the correlation time of the generated signal may be similar to the shorter one between the correlation times due to the platform and clutter dynamics.

\section{SIMULATION RESULTS}

The simulation parameters are summarized in Table 1. The parameters of correlation length, root mean square (RMS), and dielectric constant of the rough surface are found in [5]. The pulse repetition frequency (PRF) is the reciprocal of PRI. A complete signal generation is considered, including two Doppler effects. For the rest of the simulations, the platform orientation and antenna beam steering are set as $\theta_{r}=45^{\circ}, \theta_{y}=0^{\circ}, \theta_{p}=$ $0^{\circ}$, and $\theta=130^{\circ}, \varphi=0^{\circ}$, respectively. In Fig. 8, the RD maps are shown for the sum and elevation difference signals. The $\mathrm{RD}$ map is calculated by taking the fast Fourier transform (FFT) along the slow-time axis, which clearly shows the signal Doppler frequencies. The correlation time of the clutter is assumed as 0.03 seconds for an $\mathrm{X}$-band ground clutter [25]. The Doppler frequency can be exactly computed as $9,192.5 \mathrm{~Hz}$ at the center of the projected ellipse. The signal Doppler frequencies on the projected antenna footprint distributes over the wrapped Doppler frequency range $\left(-800<f_{D}<-700\right) \cup$ $\left(344.6<f_{D}<800\right)[19]$. Hence, the correct Doppler frequency

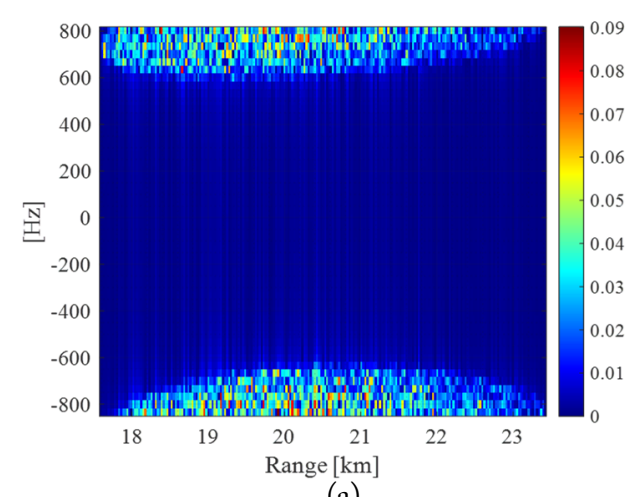

(a)

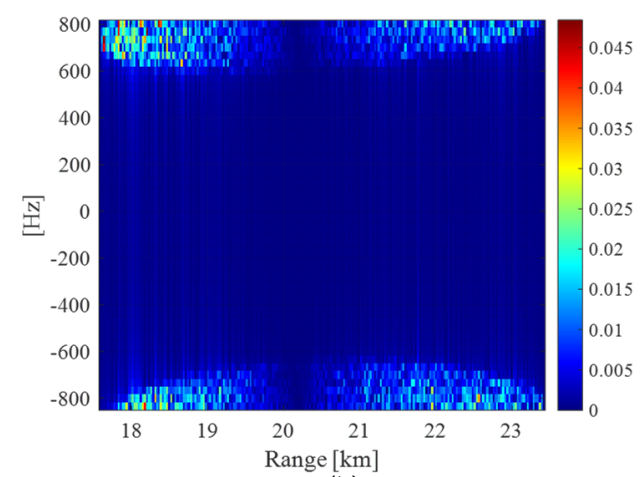

(b)

Fig. 8. RD maps of monopulse signals for $t_{c}=0.03$ seconds: (a) sum signal $\left(\Psi_{\Sigma}\right)$ and (b) elevation signal $\left(\Psi_{\triangle E L}\right)$. generation is observed in Fig. 8. In Fig. 8(b), the null of the elevation signal can be observed. In the Tx mode, only the sum pattern is transmitted, and so the Doppler frequency distribution of the difference pattern is similar to that of the sum pattern. Over the projected antenna footprint on the ground, the Doppler frequencies exit over a $500-\mathrm{Hz}$ band, which indicates that the $500-\mathrm{Hz}$ frequency band is generated due to the platform dynamics. Hence, the correlation time of the return signal due to the platform movement is around $1 / 500 \approx 0.002$ seconds $[22,24]$. Thus, two correaltion times of the ground clutter is chosen: a longer correlation time, 0.03 seconds, and a shorter one, 0.001 seconds. As explained earlier, the correlation time of the generated radar signal should be similar to the shorter one between the correlation times due to the platform and clutter dynamics. In Fig. 9, the correlation of the generated signal is computed along the slow time to produce two correlation times of the clutter. It shows that the correlation time becomes the shorter one between the two values of $t_{c}$ of the gound clutter and the platform.

Next, an AGR simulation is considered for a real radar application. Here, we use the coherent integration technique to combine the 2D time-domain signal [26]. The coherent technique truncates the $\mathrm{RD}$ map of the sum signal around the Doppler center. Also, the RD map of the elevation difference signal

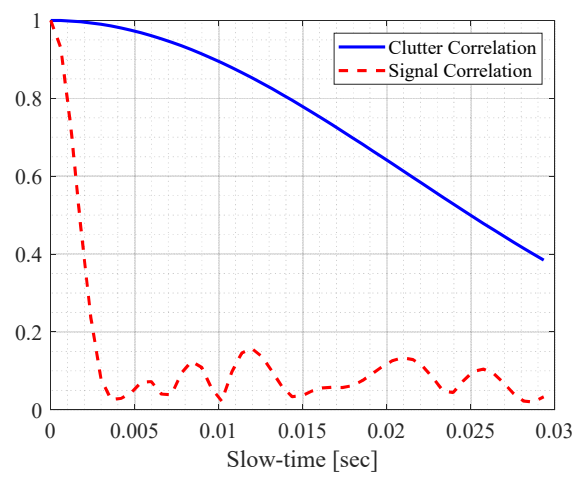

(a)

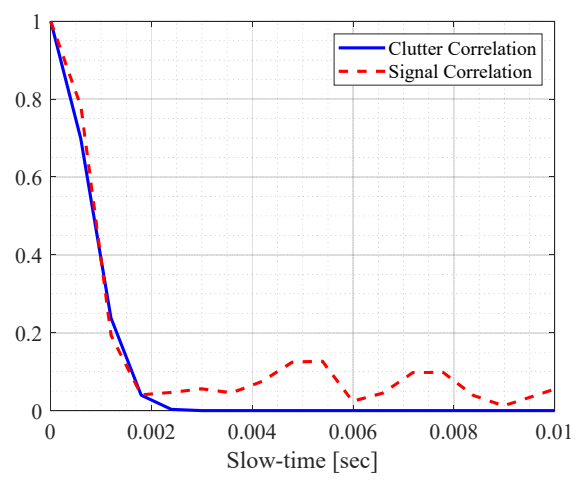

(b)

Fig. 9. Correlation of received signals: (a) $t_{c}=0.03$ seconds and (b) $t_{c}=0.001$ seconds. 


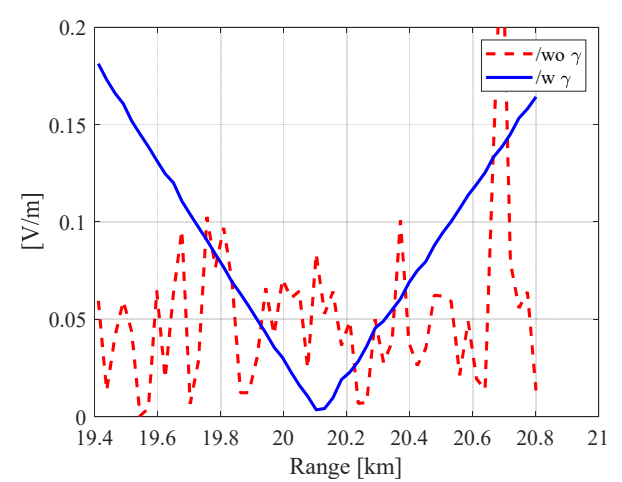

Fig. 10. Monopulse ratio for AGR simulation with and without $\gamma^{-}$ correction.

is chosen from the same range as that for the sum pattern. Then, the monopulse ratio is calculated and averaged to one ratio [14, 26]. In Fig. 10, the ratio is shown with and without a $\gamma$ correction scheme. The $\gamma$-correction scheme can retrieve the correct monopulse ratio, which enhances the accuracy of the range estimation. The null point of the monopulse ratio is around the range estimation [26]. Without the $\gamma$-correction scheme, the range estimation error is about $2.06 \%$ but with the $\gamma$-correction scheme, the error is drastically reduced to about $0.07 \%$ which is within the known error range $(<\approx 1 \%)$ of the phase-comparison monopulse AGR scheme [14].

\section{CONCLUSION}

A numerical algorithm was proposed to generate the ground return signal in a time domain for a monopulse radar system, which can accurately consider the radar operation scenario and the Doppler effects due to the movements of the platform and ground clutter. To truncate the return area, the main beam up to the HPBW is projected on the ground surface. Since the projected antenna footprint is estimated using a ray-tracing technique, the accuracy of the footprint can be very high for any radar operation scenario. To simulate the monopulse system, the sum signal is transmitted and then, using the appropriate array weights, the sum and two difference signals are synthesized. This method guarantees the correlation relationship between the sum and difference signals from an identical ground cell. The backscattering coefficient of the ground clutter is assumed to be a Gaussian random process. Thus, the Doppler effect of the ground clutter can be efficiently modeled using a correlated random number generation. For an application example, an AGR simulation is considered. The proposed signalgeneration method is applied to a monopulse AGR simulation. It is numerically shown that with the known $\gamma$-correction scheme, the signal distortion caused by rolling of the platform can be effectively removed. The range estimation error is within the known error boundary for the phase-comparison monopulse AGR scheme.

This work was supported by a grant-in-aid of Hanwha Systems (No. U-18-015).

\section{REFERENCES}

[1] M. I. Skolnik, Radar Handbook. New York, NY: McGrawHill, 1970.

[2] J. A. Malas, "F-22 radar development," in Proceedings of the IEEE 1997 National Aerospace and Electronics Conference (NAECON), Dayton, OH, 1997, pp. 831-839.

[3] N. J. Willis and H. D. Griffiths, Advances in Bistatic Radar. Raleigh, NC: SciTech Publishing Inc., 2007.

[4] S. M. Sherman and D. K. Barton, Monopulse Principles and Techniques, 2nd ed. Boston, MA: Artech House, 2011.

[5] Y. Oh, K. Sarabandi, and F. T. Ulaby, "An empirical model and an inversion technique for radar scattering from bare soil surfaces," IEEE Transactions on Geoscience and Remote Sensing, vol.30, no. 2, pp. 370-381, 1992.

[6] J. C. Curlander and R. N. McDonough. Synthetic Aperture Radar: Systems and Signal Processing. New York, NY: John Wiley \& Sons, 1991.

[7] X. M. Li, D. Z. Feng, H. W. Liu, M. D. Xing, and D. Luo, "Two-dimensional pulse-to-pulse canceller of ground clutter in airborne radar," IET Radar, Sonar \& Navigation, vol. 3, no. 2, pp. 133-143, 2009.

[8] G. Zhu, Y. Chen, and H. Yin, "Analysis of typical ground clutter statistical characteristics," in Proceedings of 2017 International Applied Computational Electromagnetics Society Symposium (ACES), Suzhou, China, 2017, pp. 1-2.

[9] A. C. Gurbuz, "Determination of background distribution for ground-penetrating radar data," IEEE Geoscience and Remote Sensing Letters, vol. 9, no. 4, pp. 544-548, 2011.

[10] Y. Zhang, H. Hu, and J. Luo, Distributed Antenna Systems: Open Architecture for Future Wireless Communications. Boca Raton, FL: Auerbach Publications, 2007.

[11] S. Sorooshyari and D. G. Daut, "Generation of correlated Rayleigh fading envelopes for accurate performance analysis of diversity systems," in Proceedings of the 14th IEEE Proceedings on Personal, Indoor and Mobile Radio Communications (PIMRC), Beijing, China, 2003, pp. 1800-1804.

[12] A. P. Szabo, "Clutter simulation for airborne pulse-Doppler radar," in Proceedings of the International Conference on Radar (IEEE Cat. No. O3EX695), Adelaide, Australia, 2003, 
pp. 608-613.

[13] B. R. Mahafza, Introduction to Radar Analysis, 2nd ed. Boca Raton, FL: CRC Press, 2017.

[14] P. K. Zwagerman, "Air-to-ground ranging using electronic roll stabilization of monopulse data," in Proceedings of the 1988 IEEE National Radar Conference, Ann Arbor, MI, 1988, pp. 151-156.

[15] A. K. Fung, Microwave Scattering and Emission Models and Their Applications. Boston, MA: Artech House, 1994.

[16] H. Laur, P. Bally, P. Meadows, J. Sanchez, B. Schaettler, E. Lopinto, and D. Esteban, "Derivation of the backscattering coefficient $\sigma 0$ in ESA ERS SAR PRI products," European Space Agency, Noordwijk, The Netherlands, ESA Document ES-TN-RE-PM-HLO9, 2002.

[17] H. Anton and R. C. Busby, Contemporary Linear Algebra. Hoboken, NJ: John Wiley \& Sons, 2003.

[18] J. J. Hiroshige and T. A. Kennedy, "Error analysis of electronic roll stabilization for electronically scanned antennas," in Proceedings of the 1991 IEEE National Radar Conference, Los Angeles, LA, 1991, pp. 71-75.

[19] B. R. Mahafza, Radar Systems Analysis and Design Using MATLAB, 3rd ed. Boca Raton, FL: CRC Press, 2013.

[20] Y. Zheng, S. M. Tseng, and K. B. Yu, "Closed-form four-

\section{Jeong-Hun Nam}

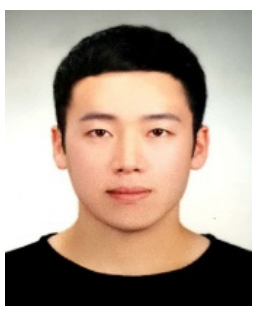

received a B.S. degree in electronic engineering from Gangneung-Wonju National University, Gangneung, Korea, in 2017. He is currently working toward his M.S. degree at the department of electronic engineering, Inha University, Incheon, Korea. His research interests include radar system modeling and radar clutter signal modeling and analysis.

\section{Jae-Won Rim}

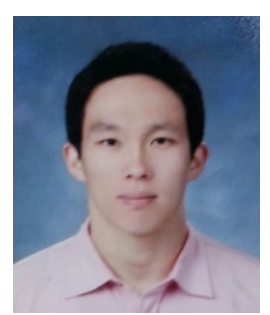

received B.S. and M.S. degrees in electronic engineering from Inha University, Incheon, Korea, in 2014 and 2016, respectively. He is currently working toward a Ph.D. at the department of electronic engineering, Inha University, Incheon, Korea. His research interests include modeling and simulation of radar systems, electronic warfare, and numerical methods in electromagnetic fields. channel monopulse two-target resolution," IEEE Transactions on Aerospace and Electronic Systems, vol. 39, no. 3, pp. 1083-1089, 2003.

[21] G. R. Curry, Radar System Performance Modeling, 2nd ed. Boston, MA: Artech House, 2004.

[22] D. Tse and P. Viswanath, Fundamentals of Wireless Communication. Cambridge, MA: Cambridge University Press, 2005.

[23] C. R. Dietrich and G. N. Newsam, "Fast and exact simulation of stationary Gaussian processes through circulant embedding of the covariance matrix," SLAM Journal on Scientific Computing, vol. 18, no. 4, pp. 1088-1107, 1997.

[24] G. V. Morris and L. Harkness, Airborne Pulsed Doppler Radar, 2nd ed. Boston, MA: Artech House, 1996.

[25] J. Xu, Y. Peng, Q. Wan, X. Wang, and X. Xia, "Doppler distributed clutter model of airborne radar and its parameters estimation," Science in China Series F: Information Sciences, vol. 47, no. 5, pp. 577-586, 2004.

[26] J. H. Nam, H. S. Lee, I. S. Koh, J. H. Song, and C. S. Bae, "Performance assessment of mono-pulse radar air-to-ground ranging algorithm for various clutter environments," The Journal of Korean Institute of Electromagnetic Engineering and Science, vol. 31, no. 7, pp. 631-639, 2020.

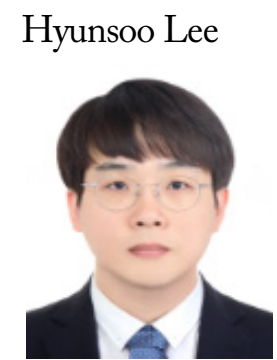

received his M.S. and Ph.D. degrees in electronics engineering from Inha University, Incheon, Korea, in 2014 and 2019, respectively. He is currently working at the agency for defense development, Daejeon, Korea, as a research engineer. His research interests include electromagnetic numerical analysis and diffraction by discontinuous wedge structure.
Il-Suek Koh

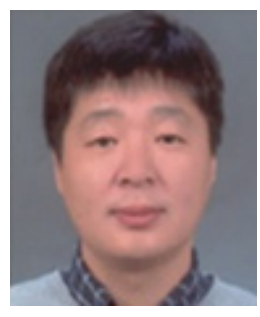

received B.S. and M.S. degrees in electronics engineering from Yonsei University, Seoul, Korea, in 1992 and 1994, respectively, and a Ph.D. degree from the University of Michigan, Ann Arbor, MI, USA, in 2002. In 1994, he joined LG electronics Ltd., Seoul, as a research engineer. Currently, he works at Inha Univerity, Incheon, Korea, as a professor. His research interests include wireless communication channel modeling and numerical and analytical methods for electromagnetic fields. 


\section{Jong-Hwa Song}

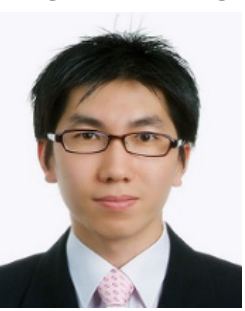

received B.S. and M.S. degrees in electronics engineering from Ajou University, Suwon, Korea, in 2005 and 2007, respectively. He received his Ph.D. from Konkuk University, Korea, in 2016. Currently, he works as a senior engineer on a Avionics Radar Team at Hanwha Systems. His main research interests are radar system design, radar signal processing, and navigation systems for airborne radar. 